\title{
BVRI CCD PHOTOMETRY OF IC 166
}

M. MANTEIGA OUTEIRO AND J.R. ACARRETA RODRIGUEZ Lab. de Astrof. Espacial y Física Fundamental, Madrid, Spain. C. MARTINEZ ROGER

Instituto de Astrofísica de Canarias, Tenerife, Spain.

AND

O. STRANIERO

Osservatorio Astronomico di Teramo, Italy.

The first results of BVRI CCD photometry of the cluster IC 166, obtained at the new telescope IAC80 at the Observatorio del Teide (Tenerife, Spain) are presented. Using the Daophot crowded field photometric package, different color-magnitude diagrams were constructed and some cluster properties (reddening, distance and age) studied.

Preliminary results: CMD and Extinction Law

The R-(R-I) diagram obtained for a total of 1792 stars shows a well defined MS, some stars on the RGB, a well populated clump of He-burning stars and also some Blue Stragglers. The CMD was fitted with an isochrone of age 0.6 Gyrs $(Y=0.27, Z=0.02)$. The value obtained for the distance modulus is $(m-M)=15.65$ and for the reddening $E(B-V)=0.80$ with a clear amount of differential reddening between the stars.

Stars on the RG clump were used to determine the differential interstellar extinction, and to estimate the reddening law in the direction of the cluster. We obtained the following extinction law:

$R=A_{V} / E(B-V)=3.014, E(V-R) / E(B-V)=0.858$ and $E(V-$ $I) / E(B-V)=1.552$ 